\title{
Constraints on the Gluon Density from Bottom Quark and Prompt Photon Production*
}

\author{
Edmond L. Berger and Ruibin Meng \\ High Energy Physics Division \\ Argonne National Laboratory \\ Argonne, IL 60439 \\ Jianwei Qiu \\ Department of Physics and Astronomy \\ Iowa State University \\ Ames, IA 50011
}

\begin{abstract}
In next-to-leading order quantum chromodynamics, gluon-gluon interactions dominate the production of bottom quarks at hadron collider energies, and gluon-quark interactions control inclusive prompt photon production at large transverse momentum in $p p$ collisions at fixed-target energies. Using such data, in conjunction with data from deep inelastic lepton scattering, we determine a new gluon density whose shape differs substantially from that derived from previous fits of data. The new set of parton densities provides a good fit to bottom quark, prompt photon, and deep inelastic data, including the most recent $\mathrm{NMC}$ and C'CFR results.
\end{abstract}

-Work supported by the U.S. Department of Energy, Division of High Energy Physics, Contract W-31-109-ENG-38 and DE-FG02-92ER40730.001.

Paper presented at the XXVI International Conference on High Energy Physics, Dallas, Texas, August 1992. 
Quantum chromodynamics is used routinely to compute expected cross sections for the production of heavy quarks and of direct (or prompt) photons in hadron interactions. ${ }^{1}$ Calculations of the short distance, hard scattering cross section have been carried out to next-to-leading order in perturbation theory. ${ }^{2,3}$ After convolution of the hard scattering cross section with parton probability densities ${ }^{4}$ determined from fits to data on other processes, cross sections are derived for the production of heavy quarks ${ }^{2}$ and of prompt photons at large transverse momentum ${ }^{3}$ in hadron-hadron scattering. Theoretical uncertainties in the final answer arise from various sources. Principal among these is the relatively poorly constrained gluon density $G(x, \mu)$, especially in the relevant region of fractional momentum $x$. For heavy quark production, $x \simeq 2 M_{T} / \sqrt{s} ; M_{T}^{2}=p_{T}^{2}+M_{Q}^{2}$, where $p_{T}$ and $M_{Q}$ are the transverse momentum and mass of the heavy quark, and $\sqrt{s}$ is the center-of-mass energy of the hadronic collision. For rrompt photon production at large transverse momentum, $x \simeq 2 p_{T} / \sqrt{s}$. In $G(x, \mu), \mu$ is the factorization scale. The sensitivity of predictions to $G(x, \mu)$ is strong. ${ }^{5}$ For example, at $\sqrt{s}=1.8 \mathrm{TeV}$, gluon-gluon subprocesses account for over $90 \%$ of the bottom quark production cross section at small values of $p_{T}$, and for over $50 \%$ even at $p_{T}=80 \mathrm{GeV}$. Existing gluon distributions fail to reproduce the $\operatorname{data}^{6}$ at $\sqrt{s}=1.8 \mathrm{TeV}$.

In this paper we use the Fermilab $\mathrm{CDF}^{6}$ and CERN $\mathrm{UA1}^{7}$ collider data on bottom quark production, the CERN WA $70^{8}$ and Fermilab E706 ${ }^{9}$ fixed-target prompt photon production data, and data from deep inelastic lepton scattering ${ }^{10-12}$ to make a new determination of the gluon density. We remark that we do not include prompt photon data at collider energies in this work. Such data include important fragmentation contributions and, for experimental reasons, require a photon isolation selection, both of which complicate the analysis. ${ }^{13}$ Our analysis is carried out entirely within the usual context of the factorization assumption and employs next-to-leading order hard scattering cross sections. ${ }^{2,3}$ Roughly stated, the ranges of $x$ probed by the different experiments are: $0.01<x<0.06$ by CDF; $0.03<x<0.16$ by UA1; $0.20<x<0.65$ by E706; and $0.35<x<0.61$ by WA70. The collider bottom quark data and the fixed target prompt photon data therefore provide support for $G(x, \mu)$ in non-overlapping but nearly contigunus ranges of $x$.

An unguided simultaneous fit to data from bottom quark production, prompt photon production, and deep inelastic lepton scattering would not be successful since there are many more data points from deep inelastic lepton scattering, with much smaller uncertainties. The strategy adopted was an iterative one: starting with an established set of parton densities, 
we forced the gluon determination by fitting first to the bottom quark and prompt photon cross sections and then, with that gluon density as a starting distribution, refitting the prompt photon, the deep inelastic, and the bottom quark cross sections together. The fits we report here were carried out in the $\overline{M S}$ factorization scheme. The collider bottom quark data points were treated as entirely independent of each other in our fits. We recognize the attendant imprecision since the experimental errors are correlated, having a common overall normalization uncertainty. Thus, our procedure assigns more weight to the collider bottom quark data than they deserve.

To determine our starting distribution, we began with the parton densities of the Morfin-Tung set B1. We retained the form of the valence quarks and the $x$ dependent shape of sea (quarks and antiquarks). The parametrization of the gluon density was altered to fit the bottom quark and prompt photon cross sections, and the normalization of sea was adjusted to satisfy the momentum sum rule. Evolution of the parton densities was carried out to two-loop level.

Our first conclusion ${ }^{5}$ is that it is not possible to fit the CDF data adequately unless $\mu$ is allowed to decrease below $\mu / M_{T}=0.5$. The CDF data prefer values of $\mu$ in the vicinity of $\mu=\frac{1}{3} M_{T}$. Settling on $\mu=\frac{1}{3} M_{T}$, we used the gluon and other densities determined from the fit to the CDF and UA1 bottom quark data as starting distributions in the simultaneous fit to the bottom quark data, prompt photon data, and data from deep inelastic lepton scattering, ${ }^{10-12}$ along with data from massive lepton pair production. ${ }^{14}$ We limited our fit to the deep inelastic data having $Q>3.16 \mathrm{GeV}$ and hadron energy $W>4 \mathrm{GeV}$. In fitting the deep inelastic data our scale choice remained $\mu=Q$, but, as remarked above, we fixed $\mu=\frac{1}{3} M_{T}$ in the case of the bottom quark data. For the prompt photon data we fixed $\mu=\frac{1}{2} p_{T}$.

A satisfactory fit was obtained to the combined data set. Comparisons with the bottom quark data are presented in Fig. 1, comparisons with the CERN WA70 and Fermilab E706 data are presented in Figs. 2 and 3, and two examples of the fit to deep inelastic data are shown in Fig. 4 and Fig. 5. In Table 1 we list the values of $\chi^{2}$ from the combined fit.

In Fig. 6 we show comparisons of our new gluon density with that from $M T$ set B1 for three values of the scale $\mu$, and in Fig. 7 we show the $x$ dependence of the ratio of the new gluon density divided by the density in Morfin-Tung set B1. We note that the bottom 
quark data require a considerable increase in $x G(x, \mu)$ in the neighborhood of $x=0.05$ for values of $\mu \simeq 5 \mathrm{GeV}$.

The qualitative features of the curve in Fig. 7 are easy to understand. The CDF bottom quark data are sensitive to gluons with values of $x=2 M_{T} / \sqrt{s}$ in the range of $0.01<x<0.06$, and the UA1 bottom quark data to gluons in the range $0.03<x<0.16$. The magnitude of the CDF data exceeds predictions based on earlier gluon densities, including set $\mathrm{B} 1$ by about a factor of 2 . Since gluon-gluon scatterin; dominates, the data require an increase in the normalization of the gluon density by roughly $\sqrt{2}$ in the relevant range of $x$. The fixed-target prompt photon data support the magnitude of $x G(x, \mu)$ for $x \geq 0.2$. In order to satisfy the momentum sum rule, the increase of the gluon density at intermediate values of $x$ must be compensated by a decrease elsewhere, resulting in the depletion observed in Fig. 7.

The enhancement of $x G\left(x, \mu_{0}\right)$ near $x=0.05$, required by the CDF data, also appears to force the new initial distribution to decrease moderately as $x$ decreases below 0.01 , in contrast to the MT B1 distribution which increases gently as $x \rightarrow 0$. We note, however, that the CDF bottom quark data with $|y|<1$ do not place any constraint on the behavior of $x G(x, \mu)$ for very small $x$, i.e., $x<10^{-3}$, and that $\mathrm{QCD}$ evolution rapidly reduces the difference as $\mu$ increases.

In this paper, we have focused on the strong constraints on the gluon density provided principally by bottom quark production data at collider energies. We have shown that a new gluon density may be derived from the CDF and UA1 data. While substantially different in character from published gluon densities, the new density is nevertheless compatible with the data on fixed target prompt photon production, deep inelastic lepton scattering, and massive lepton pair production that had been used for previous determinations of $x G(x, \mu)$. It has been observed ${ }^{15}$ that the new NMC data ${ }^{11}$ support an increase in the magnitude of the parton densities in the small $x$ region. This trend is consistent with the effect we have determined by fitting the CDF data. The longitudinal structure function $F_{L}\left(x, Q^{2}\right)$ measured in deep inelastic lepton scattering has often been recommended for ? determination of $x G\left(x, Q^{2}\right)$. Experiments at HERA should be valuable, especially in the region $0.001<x<0.1$ where the new gluon density manifests a significant increase in magnitude.

We regard the work presented here in the spirit of a proof of principle that the CDF data are constraining and can be accommodated, more than in the sririt of a strict 
determination of $x G(x, \mu)$. Important questions revolve about the role of contributions in order $\alpha_{3}^{4}$ and beyond. A more definitive quantitative fit awaits better understanding of these contributions and the greater statistical precision promised from forthcoming runs at the Tevatron.

\section{References}

1. For a recent treatment, including references to prior work, consult E. L. Berger and R. Meng, Phys. Rev. D46, 169 (1992).

2. P. Nason, S. Dawson, and R. K. Ellis, Nucl. Phys. B303, 607 (1988); B327, 49 (1989); W. Beenakker, H. Kuijf, W. L. van Neerven, and J. Smith, Phys. Rev. D40, 54 (1989); W. Beenakker, W. L. van Neerven, R. Meng, G. Schuler, and J. Smith, Nucl. Phys. B351, 507 (1991).

3. E. L. Berger and J. Qiu, to be published; P. Aurenche, R. Baier, and M. Fontannaz, Phys. Rev. D42, 1440 (1990) and references therein.

4. J. G. Morfin and W. K. Tung, Z. Phys. C52, 13 (1991); J. Kwiecinski, A. D. Martin, R. G. Roberts, and W. J. Stirling, Phys. Rev. D42, 3645 (1990); M. Diemoz, F. Ferroni, E. Longo, and G. Martinelli, Z. Phys. C39, 21 (1988).

5. E. L. Berger, R. Meng, and W-K. Tung, Phys. Rev. D46, 1895 (1992).

6. CDF Collaboration, M. J. Shochet, Fermilab-CONF-91/341-E (1991).

7. UA1 Collaboration, C. Albajar et al., Phys. Lett. B256, 121 (1991).

8. WA70 Collaboration, M. Bonesini et al., Z. Fhys. C37, 535 (1988); C38, 371 (1988).

9. E706 Collaboration, G. Alversnn et al., Phys. Rev. Lett. 68, 2584 (1992).

10. BCDMS Collaboration, A. C. Benvenuti et al., Phys. Lett. B223, 485 (1989), B237, 599 (1990); CDHSW Collaboration, J. P. Berge et al., Zeit. Phys. C49, 187 (1990).

11. NMC Collaboration, P. Amaudruz et al., CERN-PPE/92-124, submitted to Phys. Lett. 
12. CCFR Collaboration, S. R. Mishra et al., Nevis Preprint 1459, submitted for publication in Phys. Rev. Lett.

13. E. L. Berger and J. Qiu, Phys. Rev. D44, 2002 (1991).

14. E605 Collaboration, C. N. Brown et al., Phys. Rev. Lett. 63, 371 (1988).

15. A. D. Martin, R. G. Roberts, and W. J. Stirling, Rutherford Appleton Laboratory preprint RAL-92-021 (1992).

\section{DISCLAIMER}

This report was prepared as a1. account of work sponsored by an agency of the United States Government. Neither the United States Government nor any agency thereof, nor any of their employees, makes any warranty, express or implied, or assumes any legal liability or responsibility for the accuracy, completeness, or usefulness of any information, apparatus, product, or process disclosed, or represents that its use would not infringe privately owned rights. Reference herein to any specific commercial product, process, or service by trade name, trademark, manufacturer, or otherwise does not necessarily constitute or imply its endorsement, recommendation, or favoring by the United States Government or any agency thereof. The views and opinions of authors expressed herein do not necessarily state or reflect those of the United States Government or any agency thereof. 
Table 1: Values of $\chi^{2}$ from the combined fit to the prompt photon, bottom quark, deep inelastic lepton scattering and massive lepton pair production data. The top line specifies the data sets, and the second line lists the values of $\chi^{2}$ divided by the number of data points. The BCDMS data are renormalized by a factor 0.975 . The CCFR $F_{2}$ data are renormalized by a factor 0.94 . The Fermilab E605 data on massive lepton pair production are renormalized by a factor 0.8 .

\begin{tabular}{llllllllllllll}
\hline \hline UA1 & CDF & WA70 & E706 & \multicolumn{2}{c}{ BCDMS } & CDHSW & NMC & CCFR & E605 \\
& & & & $F_{2}^{H}$ & $F_{2}^{D}$ & $F_{2}$ & $x F_{3}$ & $F_{2}^{H}$ & $F_{2}^{D}$ & $0.94 \times F_{2}$ & $x F_{3}$ & $\times 0.8$ \\
\hline 0.64 & 1.15 & 0.98 & 1.36 & 0.68 & 1.08 & 0.58 & 0.51 & 1.64 & 1.15 & 2.34 & 0.88 & 0.90 \\
\hline \hline
\end{tabular}




\section{Figure Captions}

Fig. 1. The solid curves show the results of our fit to the CDF and UA1 data. They are obtained from convoluting the $O\left(\alpha_{s}^{3}\right)$ QCD hard scattering cross section with the new parton densities determined from our combined fit to the deep inelastic scattering, bottom quark, and prompt photon data.

Fig. 2. The solid curves show the results of our fit to the CERN WA70 data. They are obtained from convoluting the $O\left(\alpha_{s}^{2}\right)$ hard scattering cross section with the new parton densities.

Fig. 3. As in Fig. 2, but for the Fermilab E706 data.

Fig. 4. The solid curves show the results of our fit to the BCDMS hydrogen data set, $\mu p \rightarrow \mu X$. They are obtained from the new parton densities determined from our combined fit to the deep inelastic scattering, bottom quark, and prompt photon data.

Fig. 5. As in Fig. 4, but for the NMC deuteron data set.

Fig. 6. Solid and dashed curves show the behavior of our new gluon density and that from $M T$ set $\mathrm{B} 1$ as a function of $x$, for three values of the factorization scale $\mu$.

Fig. 7. Ratio of the new gluon density to the gluon density in Morfin-Tung set B1. The ratio is evaluated at scale $\mu=5 \mathrm{GeV}$. 


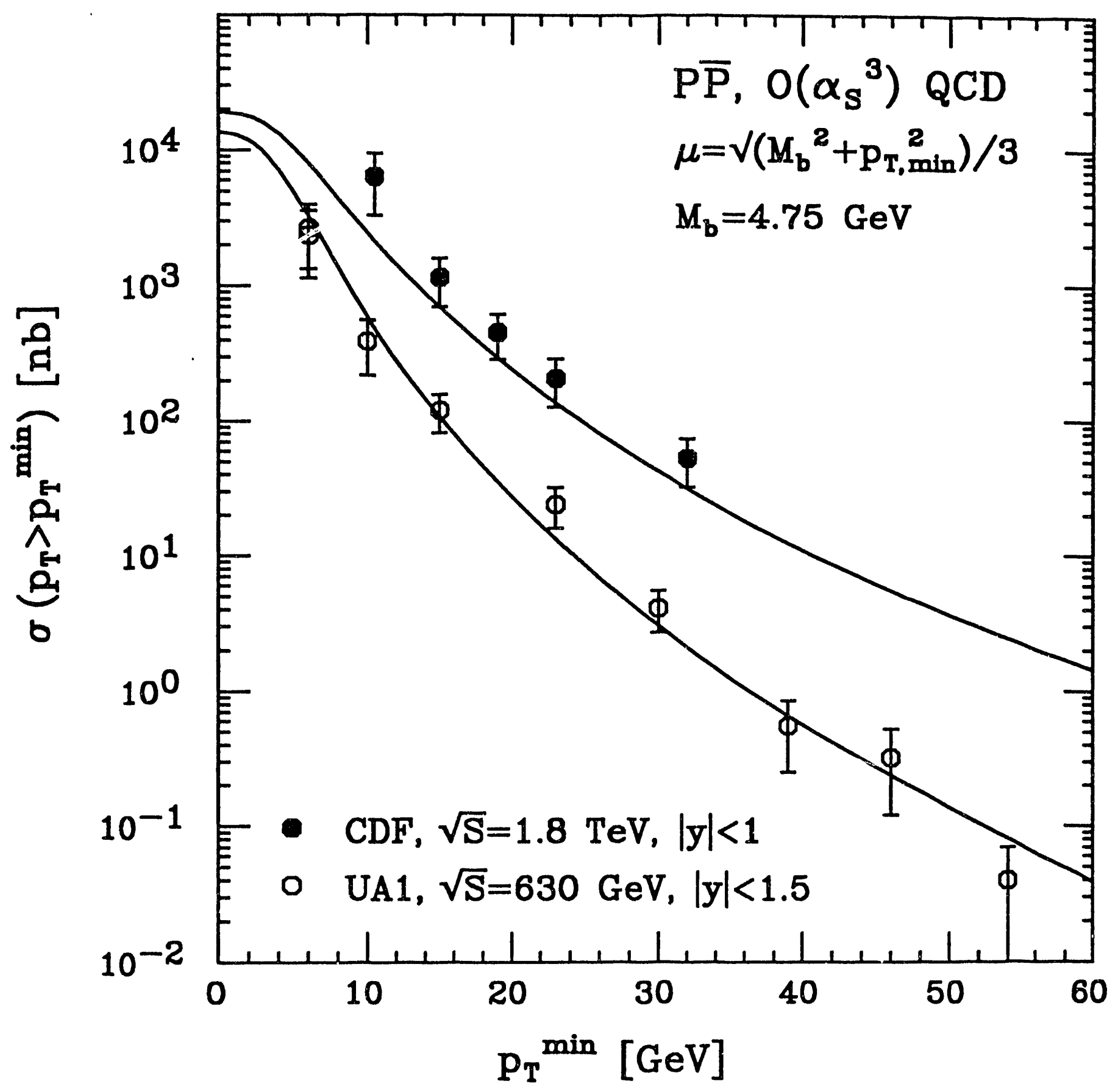

Figure 1 

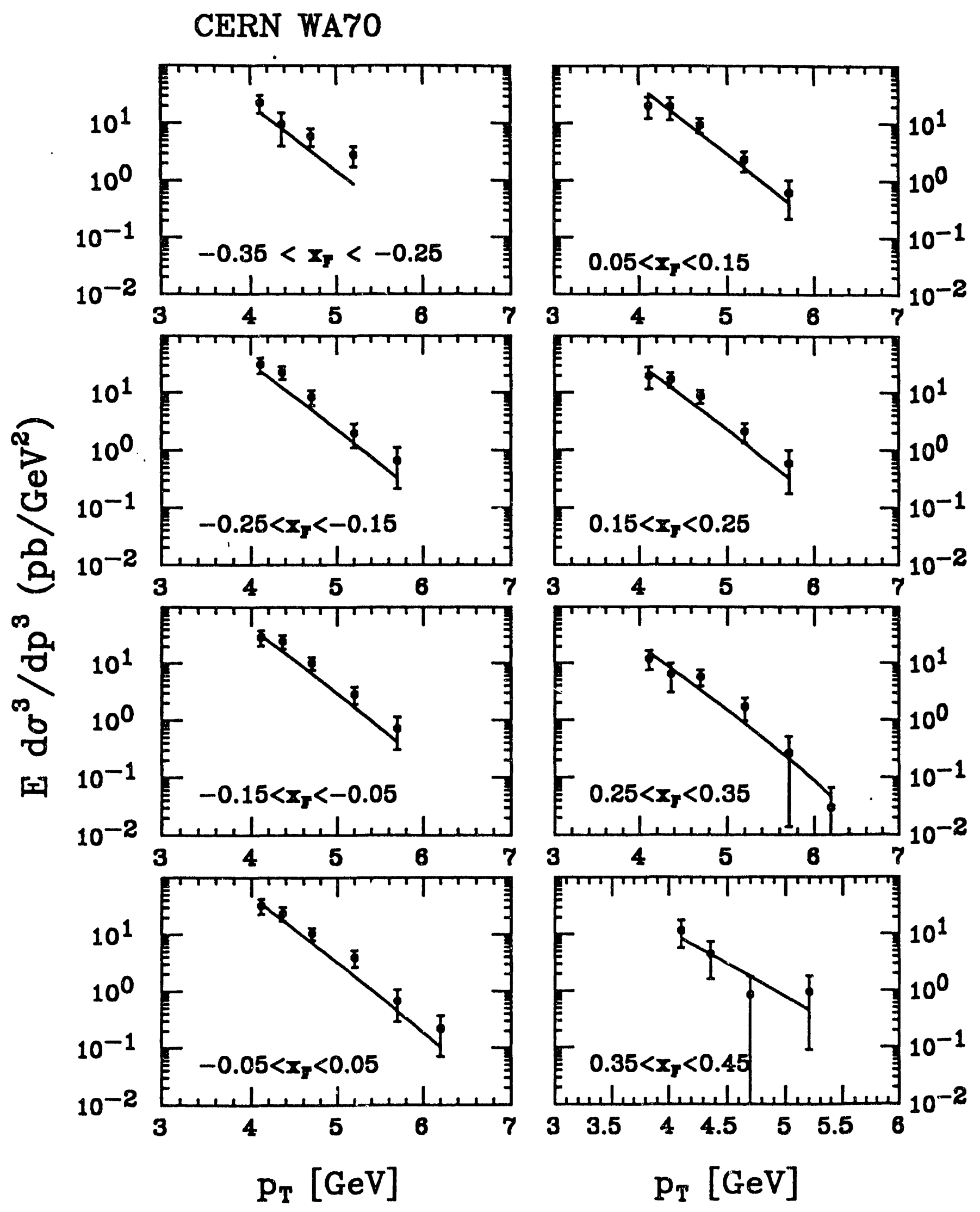

Figure 2 


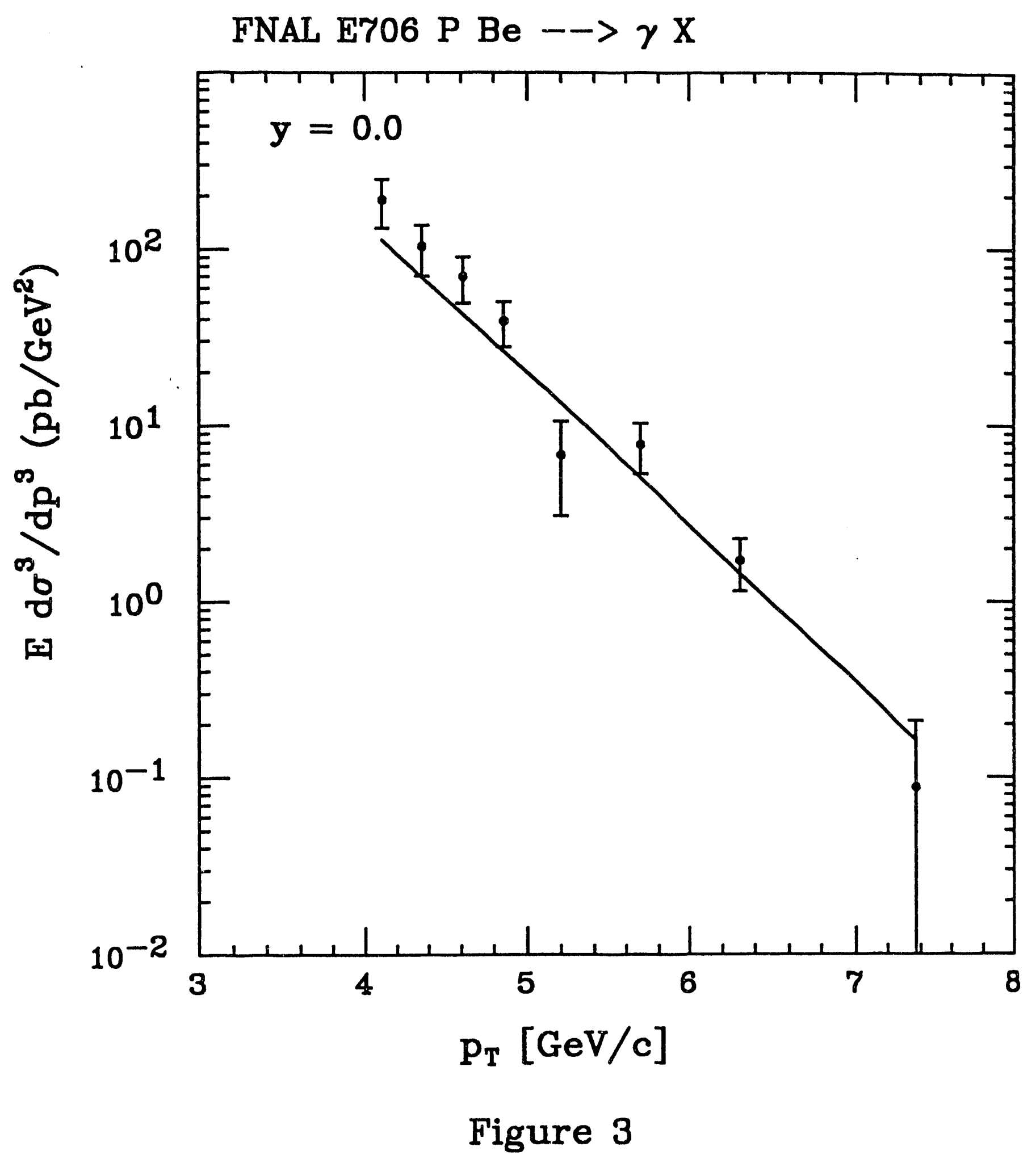




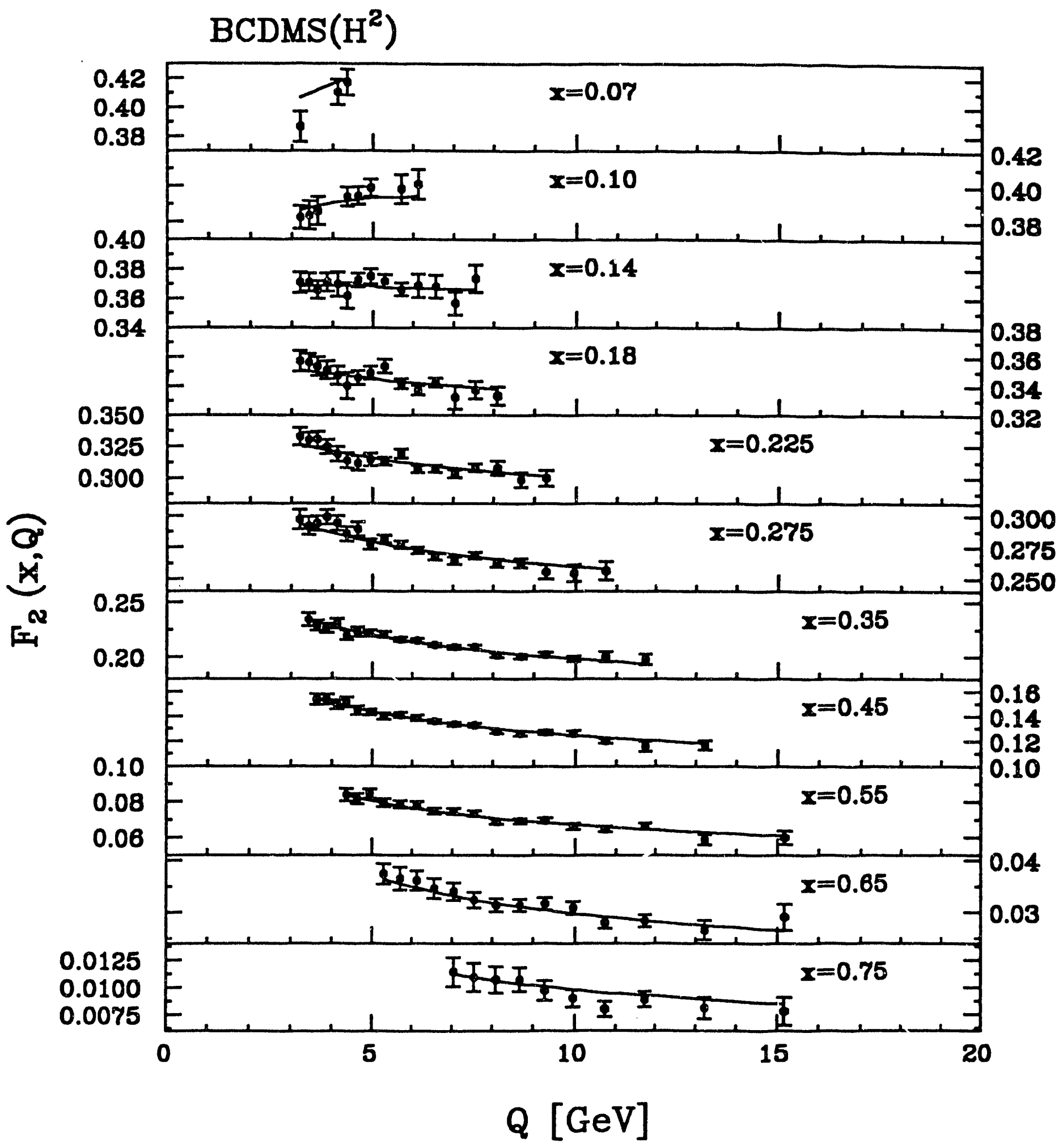

Figure 4 


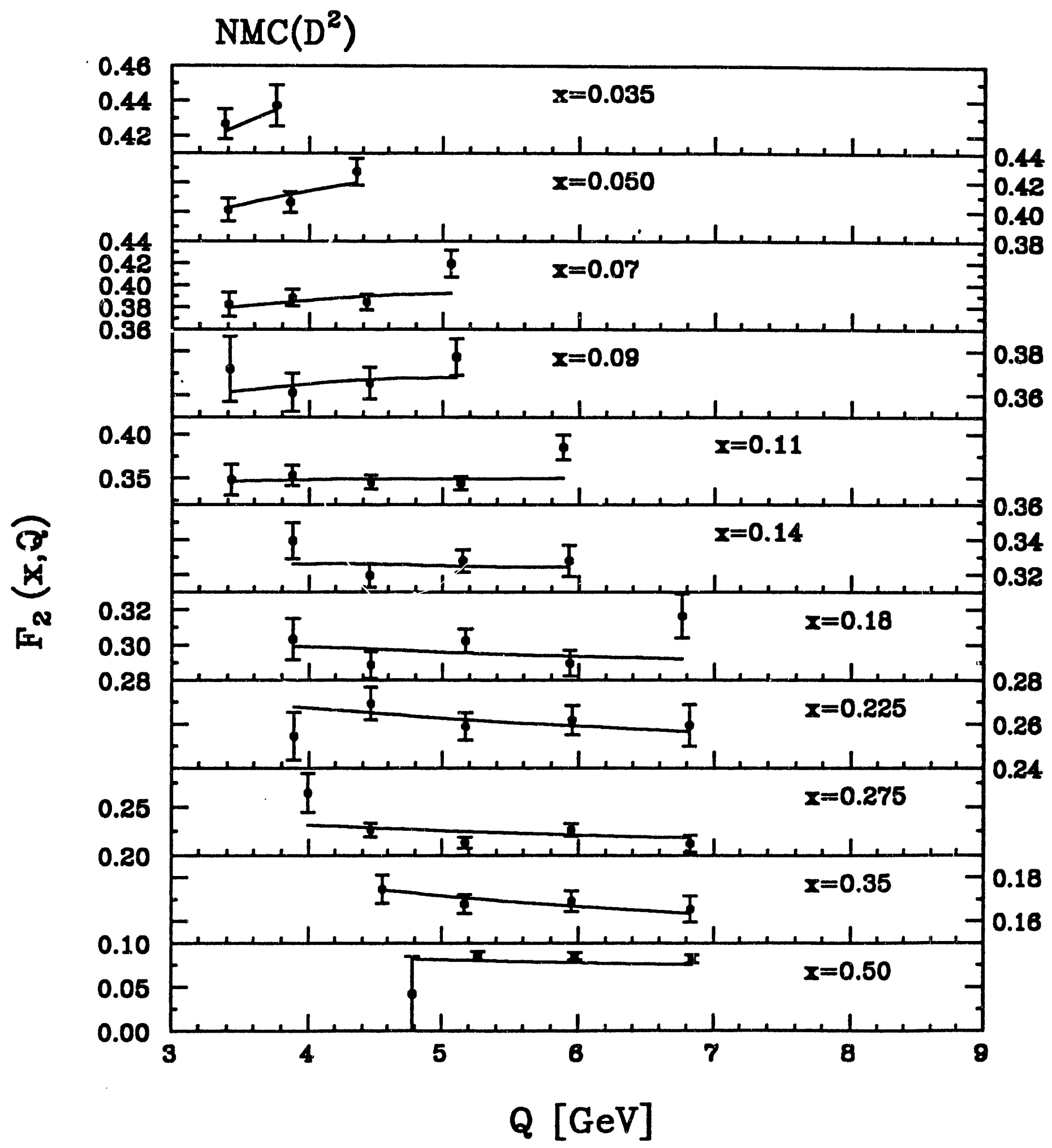

Figure 5 


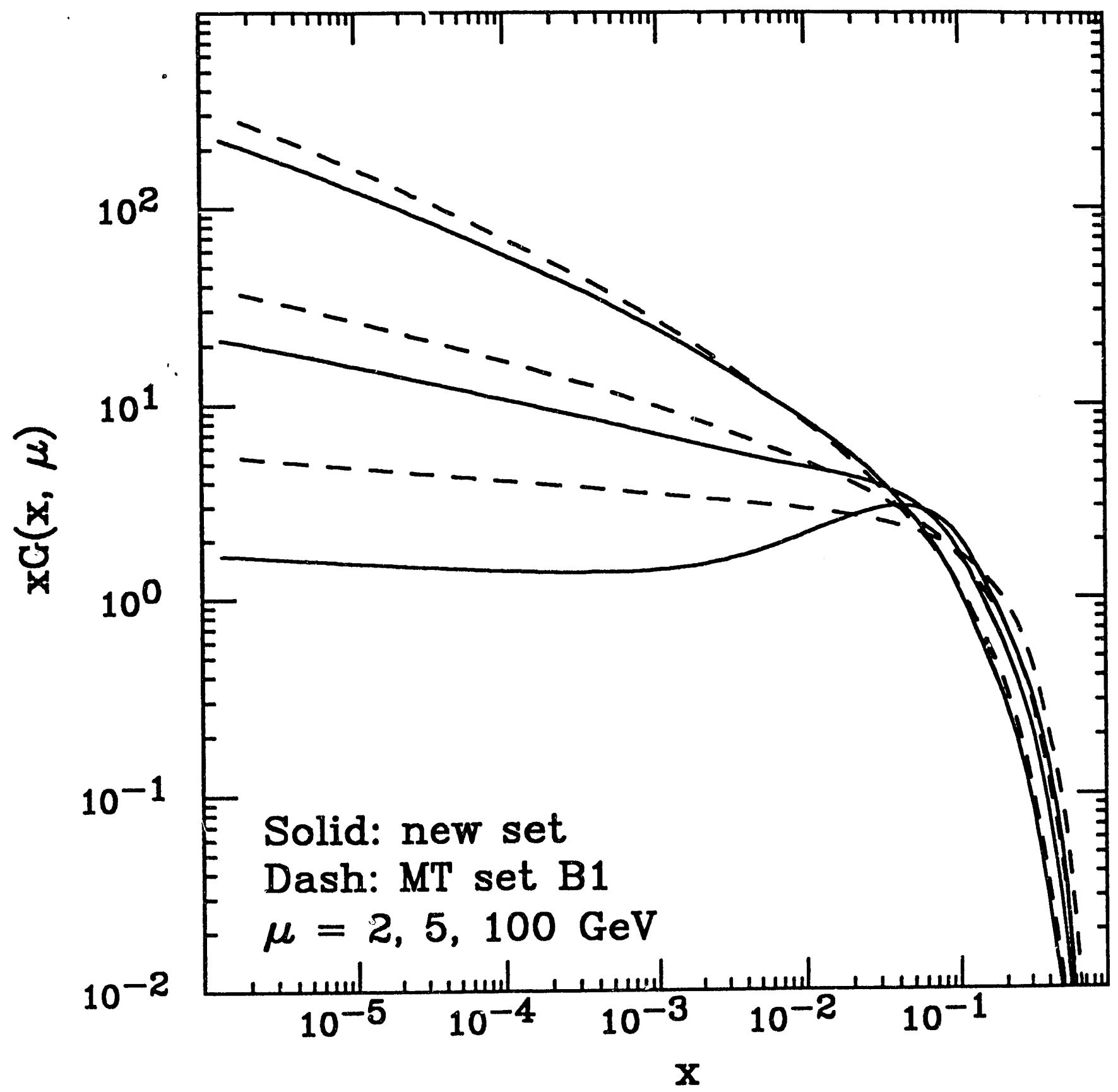

Figure 6 


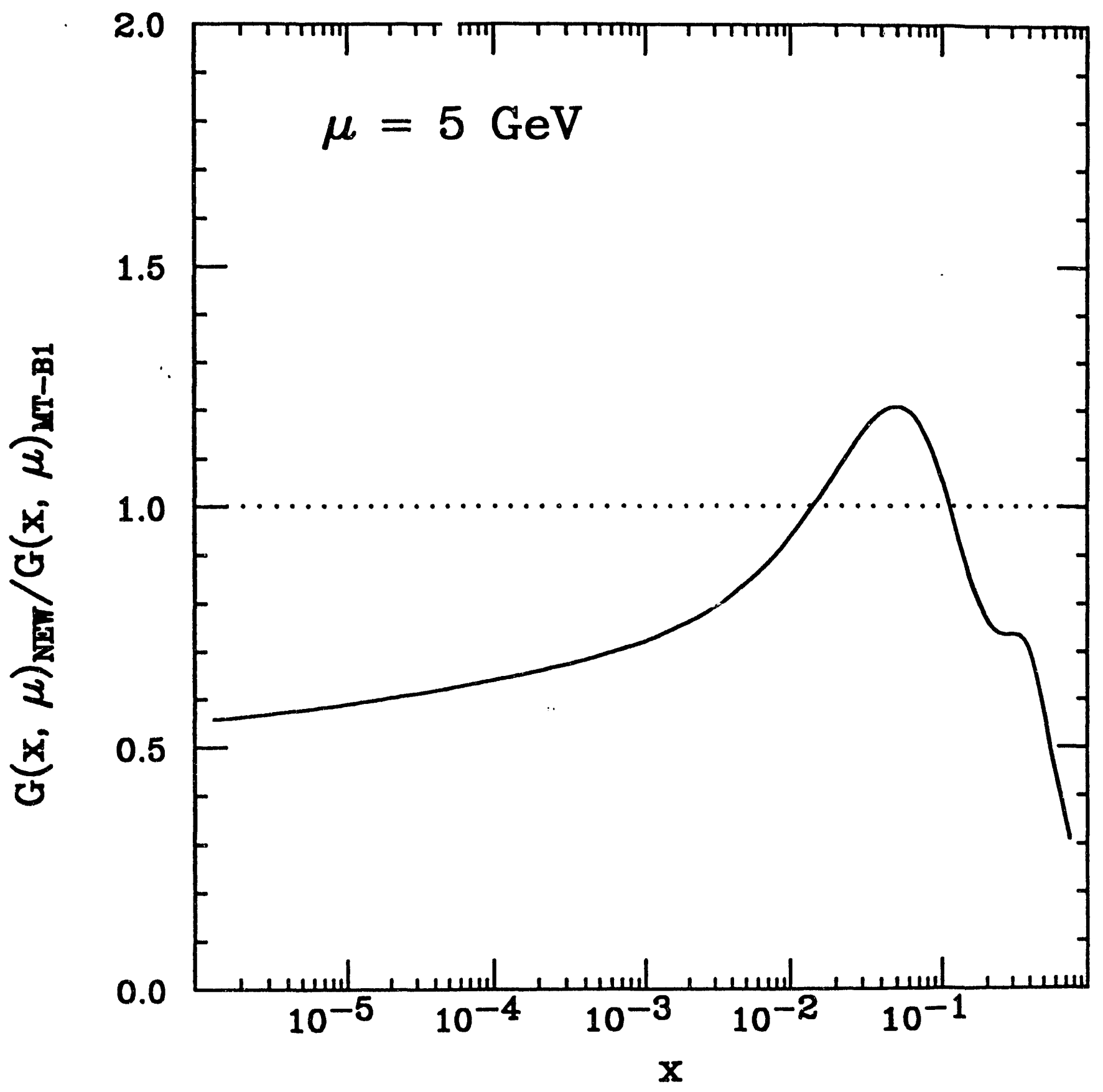

Figure 7 
DATE

FILMED

$01120 / 93$ 
